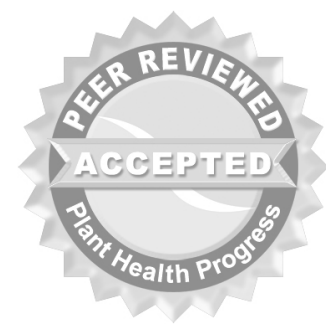

(C) 2012 Plant Management Network.

Accepted for publication 20 January 2012. Published 9 April 2012.

\title{
Compatibility of Foliar Insecticides and Soybean Cyst Nematode Bioassays
}

\author{
Chad R. Brady, Jiarui Li, Timothy C. Todd, Thomas R. Oakley \\ and Harold N. Trick, Department of Plant Pathology, Kansas State \\ University, Manhattan, KS 66502
}

Corresponding author: Harold N. Trick. hnt@ksu.edu

Brady, C. R., Li, J., Todd, T. C., Oakley, T. R., and Trick, H. N. 2012. Compatibility of foliar insecticides and soybean cyst nematode bioassays. Online. Plant Health Progress doi:10.1094/PHP-2012-0409-01-BR.

Standardized methods for assessing soybean cyst nematode (Heterodera glycines) resistance in soybeans (Glycine max) have been developed, with the assignment of resistance or susceptibility based on relative numbers of females/cysts that develop on the roots (1). However, severe infestations of greenhouse pests such as thrips, aphids, mites, and white flies can compromise bioassay results due to the impact of these aboveground pests on soybean plant health. Chemical control of insect pests in the greenhouse raises additional concerns about the direct and indirect effects that even foliar-applied insecticides, through runoff, may have on $H$. glycines development or reproduction. While the non-target effects of direct exposure to insecticides commonly used to control crop pests have been extensively studied for entomopathogenic nematodes (2), information on the compatibility of conventional insecticides and greenhouse bioassays involving root-parasitic nematodes is lacking. Nematode species can differ in their susceptibility and directional response to chemical pesticides. Studies have shown, for instance, that some pesticides can actually increase the activity of infective juveniles of entomopathogenic nematodes (3).

The objective of the present study was to investigate the effect of foliarapplied insecticides on $H$. glycines reproduction in greenhouse bioassays. In two independent trials, a total of eight different insecticides, including abamectin (Avid), bifenazate (Floramite), spiromesifen (Judo/Forbid), spinosad (Conserve), imidacloprid (Marathon II), chlorfenapyr (Pylon), hexythiazox (Hexagon), dinotefuran (Safari), and an abamectin + hexythiazox mixture, were used to treat 'Lee' soybean plants grown in D40 Deepots (Stuewe and Sons Inc., Corvallis, OR) containing soil infested with $H$. glycines HG type 7. Initial nematode population densities averaged 25,000 and 15,480 eggs $/ 100 \mathrm{~cm}^{3}$ soil for trials 1 and 2, respectively. All insecticides were applied at label recommended concentrations with a hand sprayer at a medium mist for maximum coverage. There was no attempt to shield the soil from overspray or insecticide run-off from the sprayed foliage.

In addition to the insecticide compounds used, each trial consisted of three application timing treatments: Group 1 consisted of plants that were treated with insecticides 10 days after planting to day 35; Group 2 consisted of plants that were treated with insecticides from 35 days after planting to day 70 ; and Group 3 consisted of plants that were treated with insecticides from 10 days after planting to day 70. After the first initial spray application, all treated soybean plants were sprayed with insecticides every 10 days. Non-sprayed 'Lee' plants served as controls for each group. The experimental design was a split plot with insecticide treatments as whole plots and application treatments as subplots. Whole plots were arranged in a randomized complete block with three replications. Plants were destructively harvested to obtain cyst and egg counts after 35 days for Group 1 and 70 days for Groups 2 and 3. Females and cysts were dislodged from roots with water spray and collected on a $250-\mu \mathrm{m}$-pore sieve. Cysts were mechanically ruptured to release eggs and second-stage 
juveniles. Cyst and egg counts were $\log _{10}$ transformed to minimize heterogeneity of variances prior to statistical analysis using PROC MIXED (SAS Institute Inc., Cary, NC).

Total cyst and egg counts on a per-pot basis averaged 109 and 15,117, respectively, across insecticide treatments and trials. No effects $(P \leq 0.05)$ on nematode reproduction were observed for any of the insecticide treatments, regardless of application regime. Mean numbers of $H$. glycines cysts and eggs on a per-g-dry-root-weight basis are reported for insecticide treatments in both trials in Figure 1.

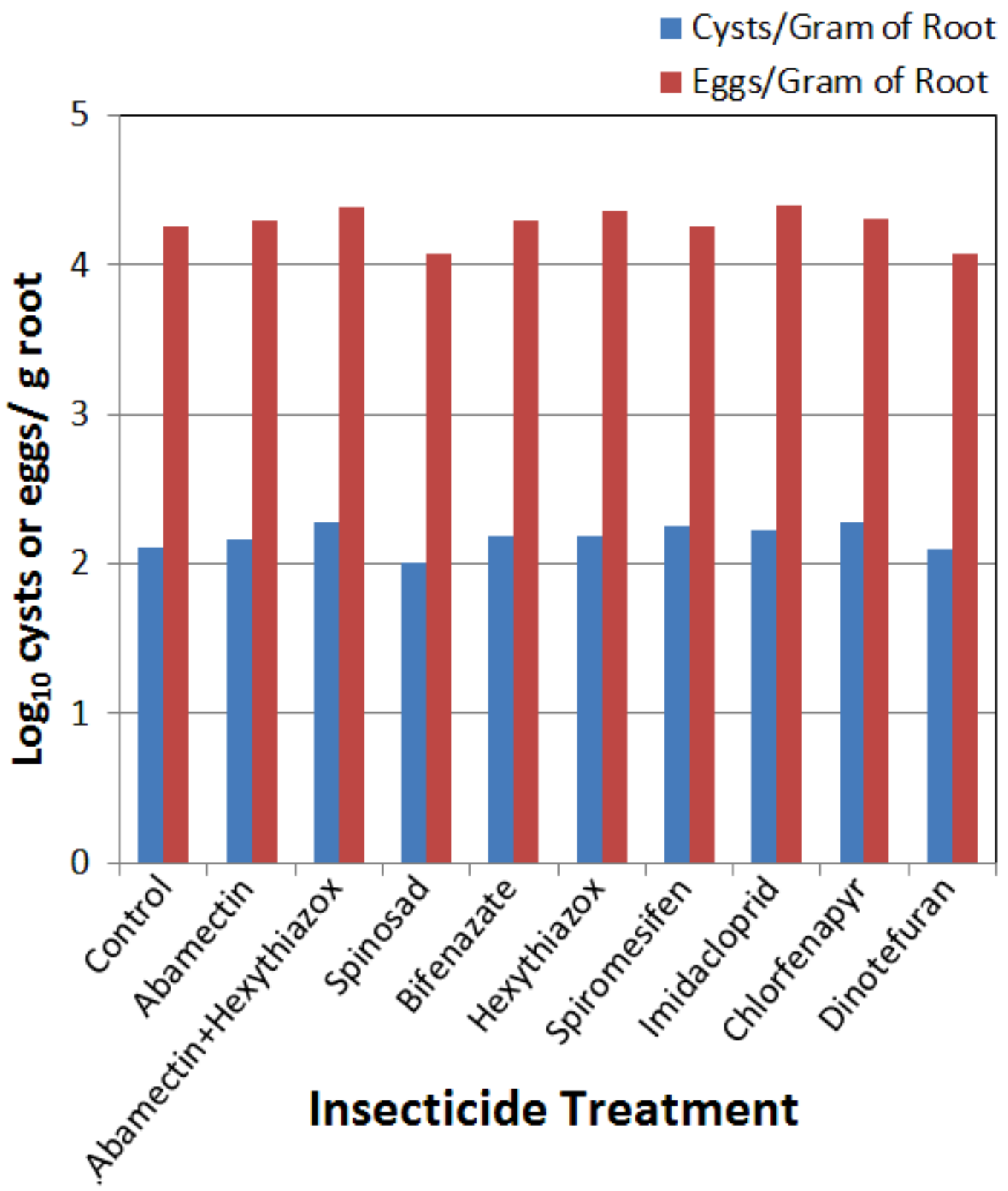

Fig. 1. Heterodera glycines population densities on insecticide-treated and non-treated Lee soybean roots. Values represent means of $\log _{10}$-transformed data. No differences among treatments were detected at $P \leq 0.05$.

In conclusion, our work demonstrated compatibility between eight different commonly used insecticides and $H$. glycines greenhouse bioassays. This result alleviates concerns of non-target effects for a range of contemporary insecticides available for control of greenhouse pests during H. glycines bioassays and 
screening trials. Better control of greenhouse insect pests may, in turn, lead to more uniform results in nematode trials.

\section{Acknowledgments}

This research was supported by the Kansas Soybean Commission and the United Soybean Board. This article is contribution no. 12-283-J from the Kansas Agricultural Experimental Station, Kansas State University, Manhattan, KS.

Literature Cited

1. Niblack, T., Tylka, G. L., Arelli, P., Bond, J., Diers, B., Donald, P., Faghihi, J., Ferris, V. R., Gallo, K., Heinz, R. D., Lopez-Nicora, H., Von Qualen, R., Welacky, T., and Wilcox, J. 2009. A standard greenhouse method for assessing soybean cyst nematode resistance in soybean: SCEo8 (standardized cyst evaluation 2008). Online. Plant Health Progress doi:10.1094/PHP-2009-0513-01-RV.

2. Head, J., Walters, K. F. A., and Langton, S. 200o. The compatibility of entomopathogenic nematode, Steinernema feltiae, and chemical insecticides for the control of the South American leafminer Liriomyza huidobrensis. BioControl 45:345-353

3. Gaugler, R., and Campbell, J. 1991. Selection for enhanced host-finding to scarab larvae (Coleoptera: Scarabaeidae) in an entomopathogenic nematode. Environ. Entomol. 20:700-706. 